\title{
On a power-type coupled system with mean curvature operator in Minkowski space
}

Zhiqian $\mathrm{He}^{1 *}$, Yanzhong Zhao ${ }^{1}$ and Liangying Miao²

\section{"Correspondence:}

zhiqianhe1987@163.com

'Department of Basic Teaching and Research, Qinghai University, Xining 810016, P.R. China

Full list of author information is

available at the end of the article

\section{Springer}

\section{Abstract}

We study the Dirichlet problem for the prescribed mean curvature equation in Minkowski space

$$
\begin{cases}\mathcal{M}(u)+v^{\alpha}=0 & \text { in } B \\ \mathcal{M}(v)+u^{\beta}=0 & \text { in } B \\ \left.u\right|_{\partial B}=\left.v\right|_{\partial B}=0 & \end{cases}
$$

where $\mathcal{M}(w)=\operatorname{div}\left(\frac{\nabla w}{\sqrt{1-|\nabla w|^{2}}}\right)$ and $B$ is a unit ball in $\mathbb{R}^{N}(N \geq 2)$. We use the index theory of fixed points for completely continuous operators to obtain the existence, nonexistence and uniqueness results of positive radial solutions under some corresponding assumptions on $\alpha, \beta$.

MSC: 34B15; 35J66

Keywords: Minkowski curvature operator; System; Positive radial solution; Uniqueness

\section{Introduction and main results}

Consider the Dirichlet problem of a quasilinear differential system of the type

$$
\begin{cases}\mathcal{M}(u)+v^{\alpha}=0 & \text { in } B \\ \mathcal{M}(v)+u^{\beta}=0 & \text { in } B, \\ \left.u\right|_{\partial B}=\left.v\right|_{\partial B}=0, & \end{cases}
$$

where $\mathcal{M}$ stands for the mean curvature operator in Minkowski space

$$
\mathcal{M}(w):=\operatorname{div}\left(\frac{\nabla w}{\sqrt{1-|\nabla w|^{2}}}\right)
$$

$B=\left\{x \in \mathbb{R}^{N}:|x|<1\right\}, N \geq 2$ is an integer.

Minkowski-curvature equations are quasilinear second-order PDEs, and there are important applications in differential geometry and the theory of relativity. Geometrically,

(c) The Author(s) 2021. This article is licensed under a Creative Commons Attribution 4.0 International License, which permits use, sharing, adaptation, distribution and reproduction in any medium or format, as long as you give appropriate credit to the original author(s) and the source, provide a link to the Creative Commons licence, and indicate if changes were made. The images or other third party material in this article are included in the article's Creative Commons licence, unless indicated otherwise in a credit line to the material. If material is not included in the article's Creative Commons licence and your intended use is not permitted by statutory regulation or exceeds the permitted use, you will need to obtain permission directly from the copyright holder. To view a copy of this licence, visit http://creativecommons.org/licenses/by/4.0/. 
these are related to maximal and constant mean curvature spacelike hypersurfaces having the property that the trace of the extrinsic curvature is zero, respectively, constant (see [12]).

It is known (see [1]) that the study of spacelike submanifolds of codimension one in the flat Minkowski space $\mathbb{L}^{N+1}\left(\mathbb{L}^{N+1}:=\left\{(x, t): x \in \mathbb{R}^{N}, t \in \mathbb{R}\right\}\right.$ endowed with the Lorentzian metric $\sum_{j=1}^{N}\left(d x_{j}\right)^{2}-(d t)^{2}$, where $(x, t)$ are the canonical coordinates in $\left.\mathbb{R}^{N+1}\right)$ with prescribed mean extrinsic curvature, can lead to the type

$$
\mathcal{M} v=H(x, v) \quad \text { in } \Omega, \quad v=0 \quad \text { on } \partial \Omega,
$$

where $\Omega$ is a bounded domain in $\mathbb{R}^{N}$ and the nonlinearity $H: \Omega \times \mathbb{R} \rightarrow \mathbb{R}$ is continuous. The existence and multiplicity of positive solutions of problem (1.2) have been discussed in the last two decades by several authors (see $[1-7,15,16,20,22,23])$ in connection with various configurations of $H$. If $\Omega$ is a bounded domain and $H$ is a bounded function defined on $\Omega \times \mathbb{R}$, Bartnik and Simon [1] proved that the problem (1.2) has a strictly spacelike solution. In particular, if $\Omega=B_{R}:=\left\{x \in \mathbb{R}^{N}:|x|<R\right\}$ with $R>0$, Bereanu, Jebelean and Torres [2,3] established some existence/nonexistence and multiplicity results for positive radial solutions of problem (1.2) via a Leray-Schauder degree argument and critical point theory. In $[6,7,15,20]$, by using the bifurcation method, the authors studied the existence, multiplicity, and the global behavior of positive solutions of problem (1.2) with $H=\lambda f(x, v)$ on the unit ball. However, to the authors' best knowledge, the study of the Dirichlet problem of a quasilinear differential system with mean curvature operator $\mathcal{M}$ seems to be in its early stages, we refer the reader to $[10-13,16,17,21]$ and the references therein. For instance, Gurban et al. [11] investigated the following two-parameter problem

$$
\begin{cases}\mathcal{M}(u)+\lambda_{1} g_{1}(|x|, u, v)=0 & \text { in } B, \\ \mathcal{M}(v)+\lambda_{2} g_{2}(|x|, u, v)=0 & \text { in } B, \\ \left.u\right|_{\partial B}=\left.v\right|_{\partial B}=0 . & \end{cases}
$$

By using the fixed-point index, they obtained the following results:

Theorem A Suppose $g_{i}:[0,1] \times[0, \infty)^{2} \rightarrow[0, \infty), i=1,2$ are continuous, quasimonotone nondecreasing with respect to both $s, t$ and satisfy for every $r \in(0,1]$,

$$
g_{i}(r, s, t)>0, \quad \forall s, t>0, \quad g_{1}(r, \xi, 0)=g_{2}(r, 0, \xi)=0, \quad \forall \xi>0
$$

and

$$
\int_{0}^{b} \tau^{N-1} g_{i}(\tau, \alpha, \alpha) d \tau>0, \quad i=1,2
$$

where $b \in(0,1), 0<\alpha<1-b$ are constants. Then, there exist $\lambda_{1}^{*}>0<\lambda_{2}^{*}$ such that for all $\lambda_{1}>\lambda_{1}^{*}$ and $\lambda_{2}>\lambda_{2}^{*}$, problem (1.3) has at least one positive radial solution. Note that (1.1) is a special case of (1.3) and Theorem A does not cover the case where $\lambda_{1}=\lambda_{2}=1$. 
In 2015, Zhang and Qi [24] studied the following system coupled by Monge-Ampère equations:

$$
\begin{cases}\operatorname{det} D^{2} u_{1}=\left(-u_{2}\right)^{\alpha} & \text { in } \Omega, \\ \operatorname{det} D^{2} u_{2}=\left(-u_{1}\right)^{\beta} & \text { in } \Omega, \\ u_{1}<0, \quad u_{2}<0 & \text { in } \Omega, \\ u_{1}=0, \quad u_{2}=0 & \text { on } \partial \Omega,\end{cases}
$$

where $\Omega$ is a ball in $\mathbb{R}^{N}, N \geq 2, \alpha>0, \beta>0$, det $D^{2} u$ stands for the determinant of the Hessian matrix $\left(\frac{\partial^{2} u}{\partial x_{i} \partial x_{j}}\right)$ of $u$. By reducing it to a system coupled by ODEs and using the fixed-point index, they obtained the existence, uniqueness results and nonexistence of radial convex solutions under some corresponding assumptions on $\alpha, \beta$.

Motivated by these studies, the main objective of this paper is to investigate the existence/nonexistence and uniqueness of positive radial solutions for system (1.1) on the unit ball $B$ mainly by the fixed-point index in a cone in the same way as in [24]. Our results are completely new and complementary to the results of [11].

We obtain:

Theorem 1.1 System (1.1) has a positive radial solution if $\alpha>0, \beta>0$ and $\alpha \beta<1$.

Theorem 1.2 Let $\alpha>0, \beta>0$ and $\alpha \beta<1$, then system (1.1) has a unique positive radial solution.

Theorem 1.3 If $\alpha>0, \beta>0$ and $\alpha \beta=1$, then system (1.1) has no positive radial solution.

This paper is organized as follows: In Sect. 2, some preliminaries are given; in Sect. 3, we obtain the main results.

\section{Preliminaries}

In order to present the existence results of positive radial solutions for system (1.1), setting $r=|x|$ and $u(|x|)=u(r), v(|x|)=v(r)$, the system (1.1) reduces to the homogeneous mixed boundary-value problem:

$$
\left\{\begin{array}{l}
\left(r^{N-1} \phi\left(u^{\prime}\right)\right)^{\prime}+r^{N-1} v^{\alpha}=0, \\
\left(r^{N-1} \phi\left(v^{\prime}\right)\right)^{\prime}+r^{N-1} u^{\beta}=0, \\
u^{\prime}(0)=u(1)=0=v(1)=v^{\prime}(0) .
\end{array}\right.
$$

By a solution of (2.1) we mean a couple of nonnegative functions $(u, v) \in C^{1}[0,1] \times$ $C^{1}[0,1]$ with $\left\|u^{\prime}\right\|<1,\left\|v^{\prime}\right\|<1$ and $r \mapsto r^{N-1} \phi\left(u^{\prime}(r)\right), r \mapsto r^{N-1} \phi\left(v^{\prime}(r)\right)$ of class $C^{1}$ on $[0,1]$, which satisfies problem (2.1). Here and below, $\|\cdot\|$ stands for the usual sup-norm on $C:=C[0,1]$.

The following lemma is a direct consequence of [18, Lemma 2.2].

Lemma 2.1 For any $u \in C([0,1],[0, \infty))$ for which $u^{\prime}(r)$ is decreasing in $[0,1]$ we have

$$
\min _{r \in\left[\frac{1}{4}, \frac{3}{4}\right]} u(r) \geq \frac{1}{4}\|u\| .
$$


Lemma $2.2([14]) \operatorname{Let} \phi(s):=s / \sqrt{1-s^{2}}$. Then, $\phi^{-1}(s)=s / \sqrt{1+s^{2}}$ and

$$
\phi^{-1}\left(s_{1}\right) \phi^{-1}\left(s_{2}\right) \leq \phi^{-1}\left(s_{1} s_{2}\right) \leq s_{1} s_{2}, \quad \forall s_{1}, s_{2} \in[0, \infty) .
$$

In particular, for $0<s_{1} \leq 1$ we have

$$
\phi^{-1}\left(s_{1} s_{2}\right) \geq s_{1} \phi^{-1}\left(s_{2}\right) .
$$

Define $P$ to be a cone in $C$ by

$$
P=\left\{u \in C: u(t)>0, t \in[0,1] \text {, and } \min _{t \in\left[\frac{1}{4}, \frac{3}{4}\right]} u(t) \geq \frac{1}{4}\|u\|\right\} .
$$

Define $P_{R}=\{u \in P:\|u\|<R\}$ for $R>0$.

For each $u \in P$, we define two solution operators $T_{i}: P \rightarrow P(i=1,2)$ as follows:

$$
\left(T_{1} u\right)(r)=\int_{r}^{1} \phi^{-1}\left(\frac{1}{t^{N-1}} \int_{0}^{t} s^{N-1} u^{\alpha} d s\right) d t
$$

and

$$
\left(T_{2} u\right)(r)=\int_{r}^{1} \phi^{-1}\left(\frac{1}{t^{N-1}} \int_{0}^{t} s^{N-1} u^{\beta} d s\right) d t .
$$

From [19], we know that each operator $T_{i}, i=1,2$ is a nonnegative concave function, this combines with Lemma 2.1 , we have $T_{i}: P \rightarrow P$ is a completely continuous operator. Define a composite operator $T=T_{1} \circ T_{2}$, which is also completely continuous from $P$ to itself. This implies from (2.2) and (2.3) that $\left(v_{1}, v_{2}\right) \in C^{1}[0,1] \times C^{1}[0,1]$ is the solution of (2.1) if and only if $v_{1}=T_{1} v_{2}, v_{2}=T_{2} v_{1}$, where $\left(v_{1}, v_{2}\right) \in P \backslash\{0\} \times P \backslash\{0\}$.

Thus, if $v_{1} \in P \backslash\{0\}$ is a fixed point of $T$, define $v_{2}=T_{2} v_{1}$, then $v_{2} \in P \backslash\{0\}$ so that $\left(v_{1}, v_{2}\right) \in C^{1}[0,1] \times C^{1}[0,1]$ solves $(1.1)$; conversely, if $\left(v_{1}, v_{2}\right) \in C^{1}[0,1] \times C^{1}[0,1]$ solves (1.1), then $v_{1}$ must be a nonzero fixed point of $T$ in $P$. Hence, our task is to search for nonzero fixed points of $T$.

Lemma 2.3 ([8]) Let E be a Banach space and $K$ a cone in E. For $r>0$, define $K_{r}=K \cap B_{r}$. Assume that $T: \bar{K}_{r} \rightarrow K$ is completely continuous such that $T x \neq x$ for $x \in \partial K_{r}=\{x \in K$ : $\|x\|=r\}$.

(i) If $\|T x\| \geq\|x\|$ for $x \in \partial K_{r}$, then $i\left(T, K_{r}, K\right)=0$.

(ii) If $\|T x\| \leq\|x\|$ for $x \in \partial K_{r}$, then $i\left(T, K_{r}, K\right)=1$.

Definition 2.4 $([9,18])$ Let $K$ be a cone in real Banach space $Y$. Let $A: K \rightarrow K$ and $u_{0}>\theta$, where $\theta$ denotes the zero element $Y$.

(i) For any $x>\theta$, there exist $\theta_{1}, \theta_{2}>0$ such that

$$
\theta_{1} u_{0} \leq A(x) \leq \theta_{2} u_{0}
$$

(ii) For any $\alpha u_{0} \leq x \leq \beta u_{0}$ and $t \in(0,1)$, there exists some $\eta>0$ such that

$$
A(t x) \geq(1+\eta) t A x .
$$

Then, $A$ is called $u_{0}$-sublinear. 
Lemma $2.5([9,18])$ An increasing and $u_{0}$-sublinear operator $T$ can have at most one positive fixed point.

\section{Proof of main results}

Proof of Theorem 1.1 By Lemma 2.2 and the definition of $T_{2}$, for each $u \in P$, we have

$$
\begin{aligned}
\left\|T_{2} u\right\| & =\int_{0}^{1} \phi^{-1}\left(\frac{1}{t^{N-1}} \int_{0}^{t} s^{N-1} u^{\beta} d s\right) d t \\
& \geq \int_{\frac{1}{4}}^{\frac{3}{4}} \phi^{-1}\left(\frac{1}{t^{N-1}} \int_{\frac{1}{4}}^{t} s^{N-1}\left(\frac{1}{4}\|u\|\right)^{\beta} d s\right) d t \\
& \geq \int_{\frac{1}{4}}^{\frac{3}{4}} \phi^{-1}\left(\left(\frac{1}{4}\|u\|\right)^{\beta}\right) \int_{\frac{1}{4}}^{t}\left(\frac{s}{t}\right)^{N-1} d s d t \\
& =\phi^{-1}\left(\left(\frac{1}{4}\|u\|\right)^{\beta}\right) \int_{\frac{1}{4}}^{\frac{3}{4}} \int_{\frac{1}{4}}^{t}\left(\frac{s}{t}\right)^{N-1} d s d t \\
& \geq \Gamma_{1} \phi^{-1}\left(\|u\|^{\beta}\right),
\end{aligned}
$$

where $\Gamma_{1}$ is a positive constant given by

$$
\Gamma_{1}=\frac{1}{4^{\beta}} \int_{\frac{1}{4}}^{\frac{3}{4}} \int_{\frac{1}{4}}^{t}\left(\frac{s}{t}\right)^{N-1} d s d t
$$

By using the same method, we can obtain

$$
\left\|T_{1} u\right\| \geq \Gamma_{1} \phi^{-1}\left(\|u\|^{\alpha}\right)
$$

Hence, we have

$$
\begin{aligned}
\|T(u)\| & =\left\|T_{1} \circ T_{2}(u)\right\| \\
& \geq \Gamma_{1} \phi^{-1}\left(\left\|T_{2} u\right\|^{\alpha}\right) \\
& \geq \Gamma_{1}^{1+\alpha} \phi^{-1}\left(\left(\phi^{-1}\left(\|u\|^{\beta}\right)\right)^{\alpha}\right) \\
& \geq \frac{1}{\sqrt{2}} \Gamma_{1}^{1+\alpha} \frac{\|u\|^{\alpha \beta}}{\left(1+\|u\|^{2 \beta}\right)^{\frac{\alpha}{2}}}
\end{aligned}
$$

which implies that

$$
\|T u\| \geq \Gamma_{2} \frac{\|u\|^{\alpha \beta}}{\left(1+\|u\|^{2 \beta}\right)^{\frac{\alpha}{2}}},
$$

where $\Gamma_{2}=\frac{1}{\sqrt{2}} \Gamma_{1}^{1+\alpha}$.

On the other hand, for each $u \in P$, we have

$$
\begin{aligned}
\left\|T_{2} u\right\| & =\int_{0}^{1} \phi^{-1}\left(\frac{1}{t^{N-1}} \int_{0}^{t} s^{N-1} u^{\beta}(s) d s\right) d t \\
& \leq \int_{0}^{1} \phi^{-1}\left(\frac{1}{t^{N-1}} \int_{0}^{t} s^{N-1}\|u\|^{\beta} d s\right) d t
\end{aligned}
$$




$$
\begin{aligned}
& \leq \int_{0}^{1} \phi^{-1}\left(\|u\|^{\beta}\right) d t \\
& \leq\|u\|^{\beta} .
\end{aligned}
$$

In the same way, we can obtain

$$
\left\|T_{1} u\right\| \leq\|u\|^{\alpha}
$$

Moreover,

$$
\|T u\| \leq\left\|T_{1} \circ T_{2}(u)\right\| \leq\|u\|^{\alpha \beta} .
$$

Now, let us consider the case of $\alpha \beta<1$. Since $\lim _{s \rightarrow 0} \frac{s^{\alpha \beta-1}}{\left(1+s^{2 \beta}\right)^{\frac{\alpha}{2}}}=\infty$, there exists $R_{1} \in(0,1)$ small enough such that for every $u \in P$ satisfying $\|u\|=R_{1}$, we have

$$
\Gamma_{2} \frac{\|u\|^{\alpha \beta-1}}{\left(1+\|u\|^{2 \beta}\right)^{\frac{\alpha}{2}}}>1 .
$$

Now, by (3.1), we have

$$
\|T u\|>\|u\| .
$$

Moreover, by (3.2), we know that there exists $R_{2}>R_{1}$, and for each $u \in P$ satisfying $\|u\|=R_{2}$ it holds that

$$
\|T u\|<\|u\| .
$$

By Lemma 2.3, we have

$$
i\left(T, P_{R_{1}}, P\right)=0, \quad i\left(T, P_{R_{2}}, P\right)=1 .
$$

Therefore, $i\left(T, P_{R_{2}} \backslash \bar{P}_{R_{1}}, P\right)=1$, which implies that $T$ has a fixed-point $u_{1} \in P_{R_{2}} \backslash \bar{P}_{R_{1}}$.

Remark 3.1 Bereanu et al. [3] studied the existence and multiplicity of positive radial solutions for the problem

$$
\mathcal{M} u+\lambda u^{q}=0 \quad \text { in } B, \quad u=0 \quad \text { on } \partial B
$$

where $q>1$. By using the Leray-Schauder degree argument and critical point theory, they obtained a sharper result: there exists $\Lambda>0$ such that problem (3.3) has zero, at least one or at least two positive radial solutions according to $\lambda \in(0, \Lambda), \lambda=\Lambda$ or $\lambda>\Lambda$. In [11], Gurban et al. investigated the existence of positive radial solutions for the Dirichlet problem of a quasilinear differential system of type

$$
\begin{cases}\mathcal{M}(u)+\lambda_{1} u^{p_{1}} v^{q_{1}}=0 & \text { in } B \\ \mathcal{M}(v)+\lambda_{2} u^{p_{2}} v^{q_{2}}=0 & \text { in } B \\ \left.u\right|_{\partial B}=\left.v\right|_{\partial B}=0, & \end{cases}
$$


where $p_{1}, q_{2}$ are nonnegative, while $q_{1}, p_{2}$ are positive exponents. By using the fixed-point index, they proved that there exist $\lambda_{i}^{*}>0$, such that for all $\lambda>\lambda_{i}^{*}$, system (3.4) has a positive radial solution $(u, v)$. We note that the relationship between $\Lambda$ and $1\left(\lambda_{i}^{*}\right.$ and 1$)$ is still uncertain. For the same reason, in this paper, if $\alpha \beta>1$, it is difficult to obtain any results in the case of $\lambda_{i}=1, i=1,2$.

Next, we can further prove that the positive radial solution obtained in Theorem 1.1 is the unique positive radial solution to problem (1.1).

Proof of Theorem 1.2 By the definitions of $T_{1}$ and $T_{2}$, it is clear that $T_{1}$ and $T_{2}$ are both increasing operators induced by $K$, where $K:=\{u \in C[0,1]: u(t) \geq 0, t \in[0,1]\}$ is a cone in $C[0,1]$. Obviously, $P \subset K$. Let $T=T_{1} \circ T_{2}$. Then, by Theorem 1.1, if we want to obtain the solution of system (1.1), we only need to prove that $T$ has at most one fixed-point in $K$. Furthermore, by Lemma 2.5 , it suffices to verify that $T: K \rightarrow K$ is $u_{0}$-sublinear for some $u_{0}$ positive in $C[0,1]$. First, let us show that $T_{2}$ satisfies the Definition 2.4(i). In fact,

$$
\begin{aligned}
\left(T_{2} u\right)(r) & =\int_{r}^{1} \phi^{-1}\left(\frac{1}{t^{N-1}} \int_{0}^{t} s^{N-1} u^{\beta} d s\right) d t \\
& \leq \int_{r}^{1} \phi^{-1}\left(\frac{1}{t^{N-1}} \int_{0}^{t} s^{N-1}\|u\|^{\beta} d s\right) d t \\
& \leq \int_{r}^{1} \phi^{-1}\left(\frac{\|u\|^{\beta}}{t^{N-1}} \frac{t^{N}}{N}\right) d t \\
& \leq \int_{r}^{1} \phi^{-1}\left(\frac{\|u\|^{\beta}}{N} t\right) d t \\
& \leq \frac{1}{2} \frac{\|u\|^{\beta}}{N}\left(1-r^{2}\right) \\
& \leq \frac{\|u\|^{\beta}}{N}(1-r) .
\end{aligned}
$$

Now, let $u_{0}=1-r, r \in[0,1]$ and $\theta_{2}=\frac{\|u\|^{\beta}}{N}$, then $T_{2}(u)(r) \leq \theta_{2} u_{0}$.

Next, let $c \in(0,1)$ be a fixed number and $\Gamma_{3}=\phi^{-1}\left(\frac{1}{c^{N-1}} \int_{0}^{c} s^{N-1} u^{\beta}(s) d s\right)$. Note that $T_{2}(u)(r)$ decreases with the variable $r$, then we obtain that

$$
T_{2}(u)(r) \geq T_{2} u(c) \geq \Gamma_{3}(1-c), \quad r \in[0, c] .
$$

By Lemma 2.2 and the fact that $r \in[c, 1]$, we have

$$
\begin{aligned}
\left(T_{2} u\right)(r) & \geq \int_{r}^{1} \phi^{-1}\left(\frac{1}{t^{N-1}} \int_{0}^{t} s^{N-1}\left(\frac{1}{4}\|u\|\right)^{\beta} d s\right) d t \\
& =\int_{r}^{1} \phi^{-1}\left(\frac{\left(\frac{1}{4}\|u\|\right)^{\beta}}{N} t\right) d t \\
& \geq \phi^{-1}\left(\frac{\left(\frac{1}{4}\|u\|\right)^{\beta}}{N}\right) \int_{r}^{1} t d t
\end{aligned}
$$




$$
\begin{aligned}
& \geq \phi^{-1}\left(\frac{\left(\frac{1}{4}\|u\|\right)^{\beta}}{N}\right) \int_{r}^{1} c d t \\
& \geq c \phi^{-1}\left(\frac{\left(\frac{1}{4}\|u\|\right)^{\beta}}{N}\right)(1-r) d t .
\end{aligned}
$$

Choose $\Gamma_{3}^{\prime}=\min \left\{\Gamma_{3}, c \phi^{-1}\left(\frac{\left(\frac{1}{4}\|u\|\right)^{\beta}}{N}\right)\right\}$. Then, we have $T_{2}(u)(r) \geq \Gamma_{3}^{\prime}(1-c)(1-r)$. Now, if we take $\theta_{1}=\Gamma_{3}^{\prime}(1-c)$, then $\theta_{1} u_{0} \leq T_{2}(u) \leq \theta_{2} u_{0}$, which satisfies Definition 2.4(i). Similarly, $T_{1}$ also satisfies Definition 2.4(i). This implies that the operator $T$ satisfies Definition 2.4(i).

Secondly, we prove that for any $\theta_{1} u_{0} \leq x \leq \theta_{2} u_{0}$ and $\xi \in(0,1)$, there exists some $\eta>0$ such that

$$
T(\xi u) \geq(1+\eta) \xi T u .
$$

From the definition of $T_{1}$ and $T_{2}$, it is easy to obtain

$$
T_{2}(\xi x) \geq \xi^{\beta}\left(T_{2} x\right), \quad T_{1}(\xi x) \geq \xi^{\alpha}\left(T_{1} x\right)
$$

Moreover, for $0<\alpha \beta<1$, there exists $\eta>0$ such that

$$
T(\xi x) \geq T_{1} \circ\left(\xi^{\beta} T_{2}(x)\right) \geq \xi^{\alpha \beta} T_{1} \circ T_{2}(x) \geq(1+\eta) \xi T x
$$

which implies that $T$ satisfies Definition 2.4(ii). Then, $T$ is $u_{0}$-sublinear and $T$ has at most one fixed-point in $K$ by Lemma 2.5. Therefore, the system (1.1) has a unique positive radial solution.

Finally, we prove the nonexistence results.

Proof of Theorem 1.3 Suppose on the contrary that $T$ has a positive fixed point $v_{0} \in P$, then $\left\|v_{0}\right\|=\left\|T v_{0}\right\|$ and $v_{0}$ is a concave function satisfying

$$
v_{0}(1)=0, \quad v_{0}(t)>0, \quad t \in[0,1) .
$$

From the proof of Theorem 1.1, we know that for any $u \in P,\left\|T_{1}(u)\right\| \leq\|u\|^{\alpha}$ and $\left\|T_{2}(u)\right\| \leq$ $\|u\|^{\beta}$. Let $u=v_{0}$, then combining this with the concavity property of $v_{0}$, we obtain that $\left\|T_{1}\left(v_{0}\right)\right\|<\left\|v_{0}\right\|^{\alpha}$ and $\left\|T_{2}\left(v_{0}\right)\right\|<\left\|v_{0}\right\|^{\beta}$. Moreover,

$$
\left\|T\left(v_{0}\right)\right\|<\left\|v_{0}\right\|^{\alpha \beta}=\left\|v_{0}\right\|,
$$

which is a contradiction. Therefore, the system (1.1) has no positive radial solution.

Acknowledgements

This work is supported by the Natural Science Foundation of Qinghai Province (2021-ZJ-957Q) and the National Natural Science Foundation of China (No. 11671322).

Funding

Not applicable.

Availability of data and materials Not applicable. 


\section{Declarations}

\section{Competing interests}

The authors declare that they have no competing interests.

\section{Authors' contributions}

$\mathrm{ZQH}$ completed the main study and wrote the manuscript, YZZ and LYM checked the proofs process and verified the calculations. Moreover, all the authors read and approved the last version of the manuscript.

\section{Author details}

'Department of Basic Teaching and Research, Qinghai University, Xining 810016, P.R. China. ${ }^{2}$ School of Mathematics and Statistics, Qinghai Nationalities University, Xining 810007, P.R. China.

\section{Publisher's Note}

Springer Nature remains neutral with regard to jurisdictional claims in published maps and institutional affiliations.

Received: 17 August 2021 Accepted: 26 October 2021 Published online: 20 November 2021

\section{References}

1. Bartnik, R., Simon, L.: Spacelike hypersurfaces with prescribed boundary values and mean curvature. Commun. Math. Phys. 87, 131-152 (1982-1983)

2. Bereanu, C., Jebelean, P., Torres, P.J.: Positive radial solutions for Dirichlet problems with mean curvature operators in Minkowski space. J. Funct. Anal. 264(1), 270-287 (2013)

3. Bereanu, C., Jebelean, P., Torres, P.J.: Multiple positive radial solutions for Dirichlet problem involving the mean curvature operator in Minkowski space. J. Funct. Anal. 265, 644-659 (2013)

4. Coelho, I., Corsato, C., Obersnel, F., Omari, P.: Positive solutions of the Dirichlet problem for the one-dimensional Minkowski-curvature equation. Adv. Nonlinear Stud. 12, 621-638 (2012)

5. Coelho, I., Corsato, C., Rivetti, S.: Positive radial solutions of the Dirichlet problem for the Minkowski-curvature equation in a ball. Topol. Methods Nonlinear Anal. 44, 23-39 (2014)

6. Dai, G.W.: Bifurcation and positive solutions for problem with mean curvature operator in Minkowski space. Calc. Var. Partial Differ. Equ. 55(4), Art. 72 (2016), 17 pp.

7. Dai, G.W.: Bifurcation and nonnegative solutions for problem with mean curvature operator on general domain Indiana Univ. Math. J. 67, 2103-2121 (2018)

8. Deimling, K.: Nonlinear Functional Analysis. Springer, Berlin (1985)

9. Guo, D., Lakshmikantham, V.: Nonlinear Problems in Abstract Cones. Academic Press, Orlando (1988)

10. Gurban, D., Jebelean, P.: Positive radial solutions for systems with mean curvature operator in Minkowski space. Rend. Ist. Mat. Univ. Trieste 49, 245-264 (2017)

11. Gurban, D., Jebelean, P.: Positive radial solutions for multiparameter Dirichlet systems with mean curvature operator in Minkowski space and Lane-Emden type nonlinearities. J. Differ. Equ. 266, 5377-5396 (2019)

12. Gurban, D., Jebelean, P., Serban, C.: Nontrivial solutions for potential systems involving the mean curvature operator in Minkowski space. Adv. Nonlinear Stud. 17, 769-780 (2017)

13. Gurban, D., Jebelean, P., Serban, C.: Non-potential and non-radial Dirichlet systems with mean curvature operator in Minkowski space. Discrete Contin. Dyn. Syst. 40(1), 133-151 (2020)

14. He, Z.Q., Miao, L.Y.: Uniqueness and multiplicity of positive solutions for one-dimensional prescribed mean curvature equation in Minkowski space. AIMS Math. 5, 3840-3850 (2020)

15. He, Z.Q., Miao, L.Y.: S-Shaped connected component of positive solutions for a Minkowski-curvature Dirichlet problem with indefinite. Bull. Iranian Math. Soc. (2021, in press). https://doi.org/10.1007/s41980-020-00512-4

16. He, Z.Q., Miao, L.Y.: Positive radial solutions for Dirichlet problems involving the mean curvature operator in Minkowski space. Int. J. Nonlinear Sci. Numer. Simul. (2021, in press). https://doi.org/10.1515/ijnsns-2020-0117

17. He, Z.Q., Miao, L.Y.: Multiplicity of positive radial solutions for systems with mean curvature operator in Minkowski space. AIMS Math. 6, 6171-6179 (2021)

18. Hu, S.C., Wang, H.Y.: Convex solutions of boundary value problems arising from Monge-Ampère equations. Discrete Contin. Dyn. Syst. 16, 705-720 (2006)

19. Liang, Z.T., Duan, L., Ren, D.D.: Multiplicity of positive radial solutions of singular Minkowski-curvature equations. Arch. Math. (Basel) 113, 415-422 (2019)

20. Ma, R.Y., Gao, H.L., Lu, Y.Q.: Global structure of radial positive solutions for a prescribed mean curvature problem in a ball. J. Funct. Anal. 270(7), 2430-2455 (2016)

21. Ma, R.Y., He, Z.Q.: Positive radial solutions for Dirichlet problem of quasilinear differential system with mean curvature operator in Minkowski space. J. Fixed Point Theory Appl. 23, Paper No. 9 (2021), 12 pp

22. Pei, M.H., Wang, L.B.: Multiplicity of positive radial solutions of a singular mean curvature equations in Minkowski space. Appl. Math. Lett. 60, 50-55 (2016)

23. Pei, M.H., Wang, L.B.: Positive radial solutions of a mean curvature equation in Lorentz-Minkowski space with strong singularity. Appl. Anal. 99, 1631-1637 (2020)

24. Zhang, Z., Qi, Z.: On a power-type coupled system of Monge-Ampère equations. Topol. Methods Nonlinear Anal. 46, 717-729 (2015) 